\title{
Ventral Hernia After Hand-Assisted Laparoscopic Nephrectomy
}

\author{
David Radvinsky, MD, Paul Chung, MD, Michael Kennedy, MD, Koby Herman, BS, \\ Gainosuke Sugiyama, MD, FACS \\ Department of Surgery, SUNY Downstate University Medical Center, Brooklyn, New York, USA (Drs Radvinsky, Chung, \\ Kennedy, and Sugiyama). \\ SUNY Downstate College of Medicine, Brooklyn, NY (Herman).
}

\begin{abstract}
Introduction: The incidence of incisional hernias after hand-assisted laparoscopic surgery (HALS) ranges from 3 to $10 \%$. Robotic-assisted ventral hernia repair is technically feasible and gaining popularity as an acceptable alternative to open repair.
\end{abstract}

Case Report: We report a case of a robot-assisted repair for an incisional hernia from a hand-assist port site in a 50-year-old man after a hand-assisted laparoscopic nephrectomy (HALN).

Conclusion: We present a novel approach for recreating the anterior abdominal wall using the robotic platform.

Key Words: Hernia, Robotic, Ventral, Pre-peritoneal.

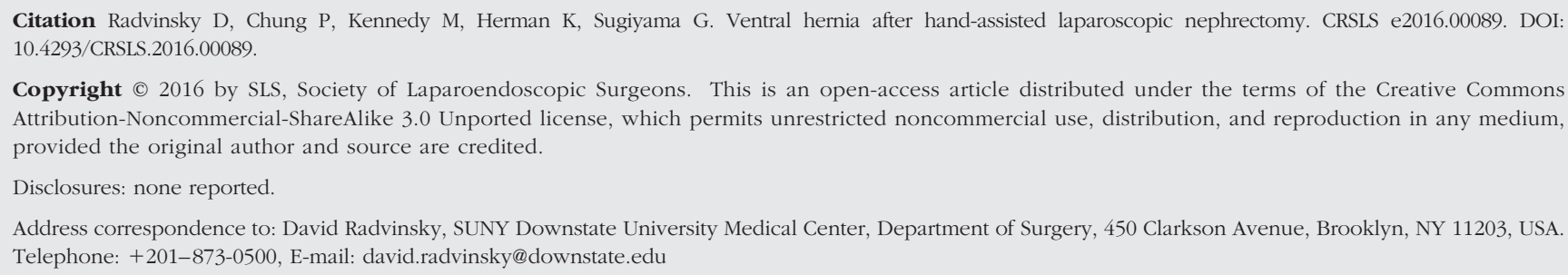

Copyright (c) 2016 by SLS, Society of Laparoendoscopic Surgeons. This is an open-access article distributed under the terms of the Creative Commons Attribution-Noncommercial-ShareAlike 3.0 Unported license, which permits unrestricted noncommercial use, distribution, and reproduction in any medium, provided the original author and source are credited.

Disclosures: none reported.

Address correspondence to: David Radvinsky, SUNY Downstate University Medical Center, Department of Surgery, 450 Clarkson Avenue, Brooklyn, NY 11203, USA. Telephone: +201-873-0500, E-mail: david.radvinsky@downstate.edu

\section{INTRODUCTION}

Violation of the fascial integrity of the anterior abdominal wall with hand-assisted laparoscopic surgery increases the risk of hernia formation. The incidence of incisional hernia after hand-assisted laparoscopic surgery (HALS) has been quoted as ranging from 3 to $10 \%$, falling between standard laparoscopic surgery and midline laparotomy..$^{1,2}$ Primary repair of this form of hernia is particularly difficult as a result of the attenuated fascia created by the herniation. Nonmidline HALS incisional hernias are a surgical problem of great complexity, but to date, little information on repair of this problem is available. Although technically challenging, repair of this condition is feasible with a laparoscopic approach and has been described with intraperitoneal and preperitoneal mesh placement.

The robotic platform allows surgeons to operate with a 3-dimensional view, perform complex wristed movements with improved ergonomics, and accomplish com- plicated procedures with more precision, flexibility, and control, compared with more conventional techniques. Robot-assisted laparoscopic ventral hernia repair allows surgeons to safely perform a lysis of adhesions, repair hernia defects primarily, create peritoneal flaps for preperitoneal mesh placement, and suture in place an intraperitoneal mesh without having to use transmuscular and transfacial sutures. We report a case of a robotic-assisted repair of a hand-assist site incisional hernia.

\section{CASE REPORT}

A 50-year-old man, body mass index (BMI) of 35, with a history of HALN for complications related to recurrent nephrolithiasis developed discomfort at the hand assist site in the right lower quadrant of his abdomen. The patient was referred to our clinic for evaluation of a ventral hernia and possible operative intervention. On physical examination, a hernia defect was palpable on the 


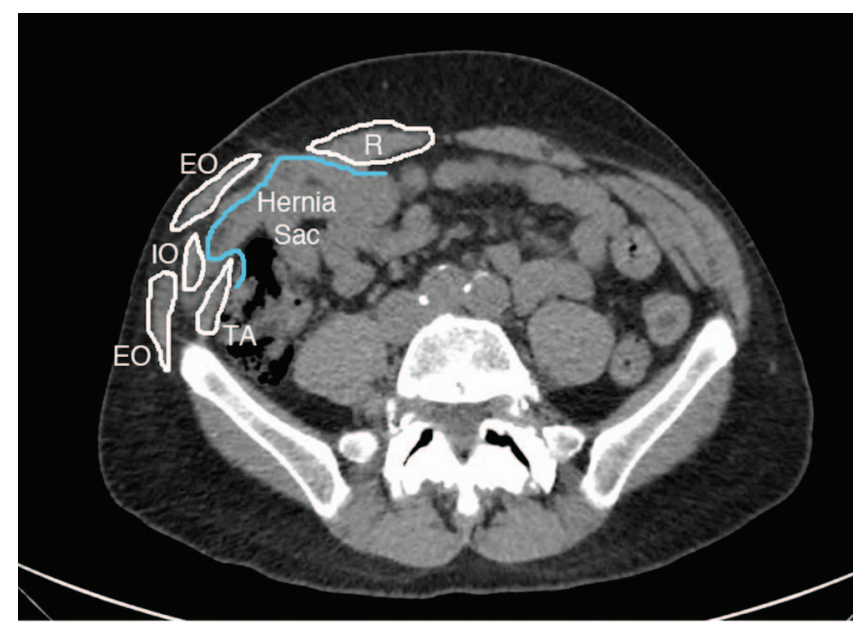

Figure 1. CT scan findings suggestive of hernia at the prior hand-assist port site, with small bowel contents, but without evidence of obstruction or strangulation. R, rectus muscle; EO, external oblique muscle; IO internal oblique muscle; TA transverse abdominis muscle.

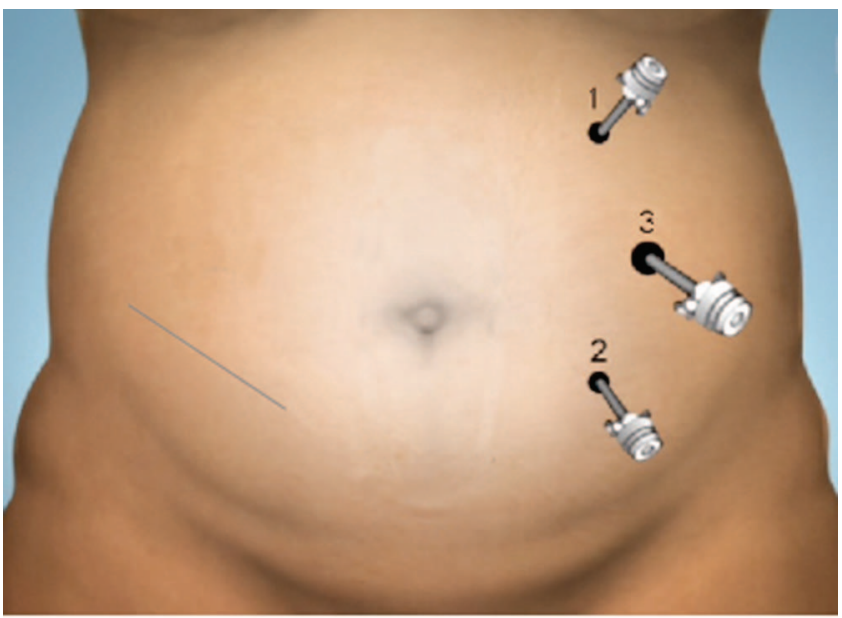

Figure 2. Port placement in relation to the hand-assist incision site.

lateral aspect of his right lower quadrant incision. A computed tomographic scan of the abdomen and pelvis revealed omentum and small bowel contents within the hernia sac, without evidence of obstruction or strangulation (Figure 1).

The patient was brought to the operating room for elective robot-assisted laparoscopic ventral hernia repair. He was placed supine on the operating room table with both arms tucked and right side up at $\sim 30^{\circ}$. The abdomen was entered in the left upper quadrant with an Optiview technique (Optiview, Jacksonville, Florida, USA). Under direct

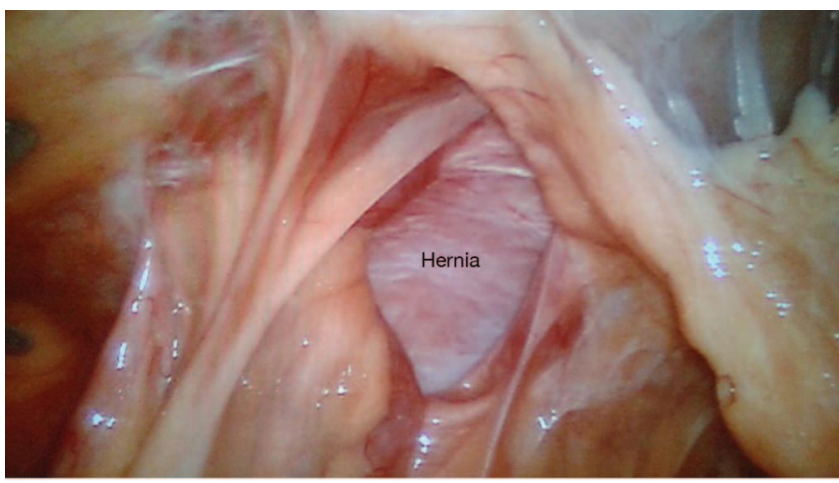

Figure 3. Intra-abdominal inspection of the hand-assist site hernia, once the robot was docked. The small-bowel contents were reduced with initial insufflation. Adhesions of the small bowel to the perimeter of the hernia were reduced with adhesiolysis.

vision, 2 additional 10-mm ports were placed laterally in the left mid abdomen and left lower quadrant (Figure 2). Inspection of the abdomen showed extensive adhesions surrounding the hernia defect, which included multiple loops of small bowel tethered to the anterior abdominal wall (Figure 3). After extensive adhesiolysis, the contents of the hernia were reduced, revealing an $\sim 6 \times 6-\mathrm{cm}$ defect. The edges of the external and internal oblique muscles were dissected, and the hernia defect was closed primarily with a running absorbable barbed sutures (Figure 4). Peritoneal flaps were created by dissection of the transversalis fascia from the internal oblique to allow a preperitoneal mesh placement. An $11 \times 11$-cm composite mesh composed of polypropylene and an absorbable hydrogel barrier was placed into the preperitoneal space (Figure 5). The mesh was secured with a circumferential absorbable running suture, resulting in $3 \mathrm{~cm}$ of peritoneum overlap from the edge of the mesh circumferentially (Figure 6). The fascia was closed at all port sites with a 0 Vicryl suture, and the skin was closed with running 4-0 Monocryl at all port sites. Estimated blood loss was minimal. Total operative time was $190 \mathrm{~min}$, time on the robotic console was 146 minutes, and docking time was 8 minutes. An assistant was at the patient's side during the entire course of the procedure.

The postoperative clinical course was uneventful. The patient was discharged home the following morning. He has been seen for routine follow-up visits in the office and showed no adverse events 30 days after surgery.

\section{DISCUSSION}

Ventral incisional hernia is a common complication after abdominal surgery. Although the increase in the use of lapa- 


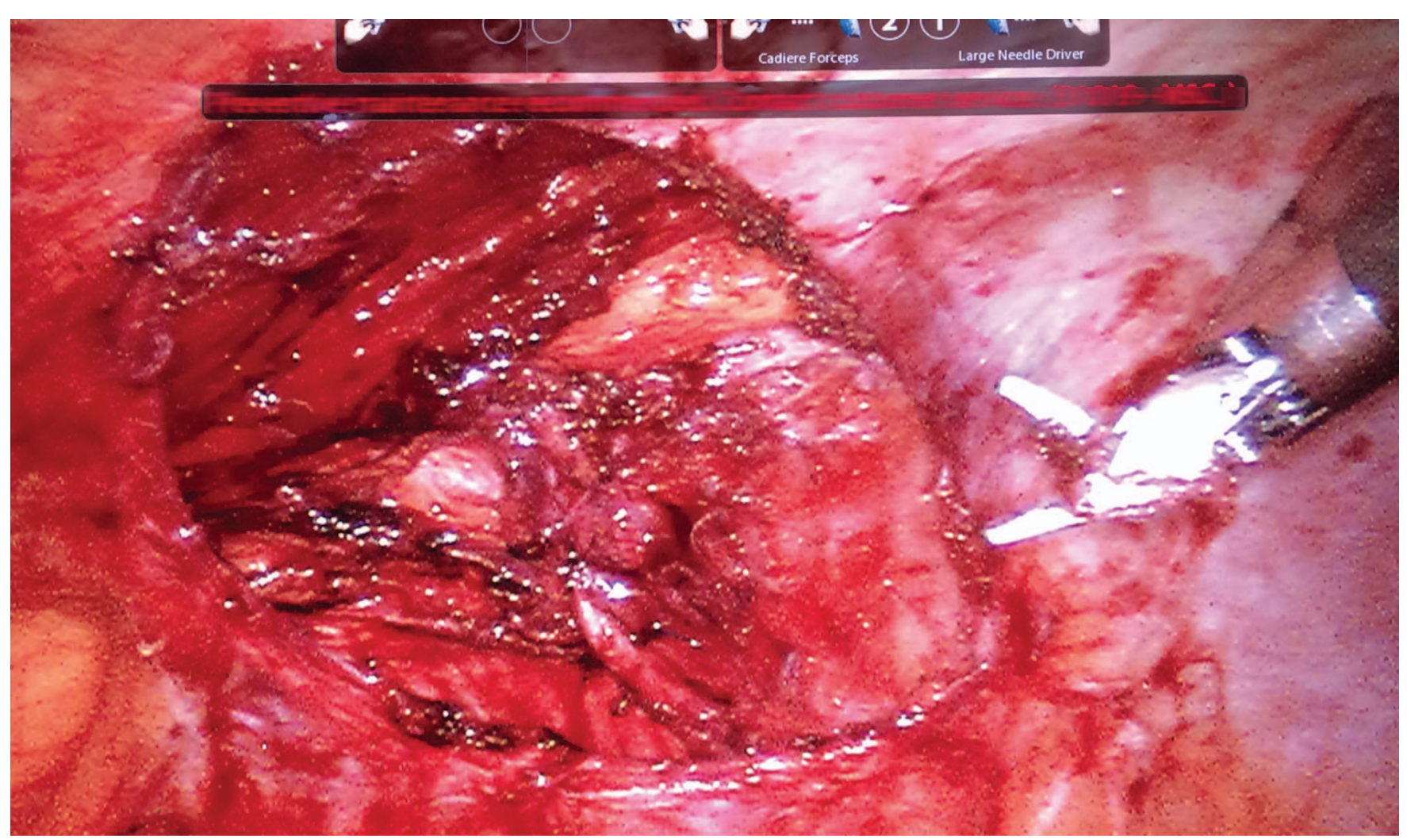

Figure 4. After adhesiolysis, the external and internal oblique muscles were dissected from the peritoneum, and the hernia defect was repaired primarily before mesh placement.

roscopic surgery has permitted a marked decrease in the rate of incisional hernia, this approach does not completely prevent the complication. Extraction sites and hand-assist sites require a larger incision compared to standard laparoscopic incisions. The overall incidence of incisional hernia after hand-assisted laparoscopic surgery is 3 to $10 \%$ and varies by location in relation to the midline. ${ }^{1,2}$

In a retrospective analysis, Troxel and $\mathrm{Das}^{4}$ reviewed 50 patients who underwent HALN and reported on the postoperative incidence of incisional hernias. Of the $50 \mathrm{pa}-$ tients, incision hernias developed in 6\% (3 patients). They concluded that such hernias have a multifactorial etiology, including patient's BMI, comorbidities, smoking status, and functional status, but that obese patients are at higher risk. They also stated that interrupted closure of the incision was superior to a running polydioxanone suture (PDS) and furthermore, with the adoption of this technique, they had not experienced any additional hernias. They did not offer suggestions for repair, however. A recent retrospective analysis of the treatment of flank and lateral abdominal wall hernias advocates creating musculofascial flaps and performing a primary nonbridged mesh repair to recreate anatomical congruity. ${ }^{5}$ Another prospective review of kidney transplant recipients with flank hernias described a large posterior component separation with transverse abdominis muscle release with mesh placement in the retromuscular plane in a sublay fashion. ${ }^{6}$ Both groups reported decreased recurrence rates 3.4 and $9 \%$, respectively, compared to the $11.4 \%$ reported by Sauerland et al. 7 in their comparison of open to laparoscopic repair of primary ventral hernias.

SAGES provides evidence-driven guidelines for laparoscopic repair of ventral hernias. ${ }^{8}$ Multiple sources have supported a tension-free primary closure of the hernia defect with mesh reinforcement, citing a reduced seroma rate, reduced recurrence rate, and improved abdominal wall integrity and contour. A minimum of $3-\mathrm{cm}$ and maximum of 5-cm overlap of mesh is recommended to reduce tension at the fixation points and provide adequate coverage. The use of suture fixation over tacking has been shown to be more cost effective, with less postoperative pain and a quicker return to activity with a comparable recurrence rate. Nonmidline ventral hernias are rarer than midline ventral hernias, and their surgical management is 


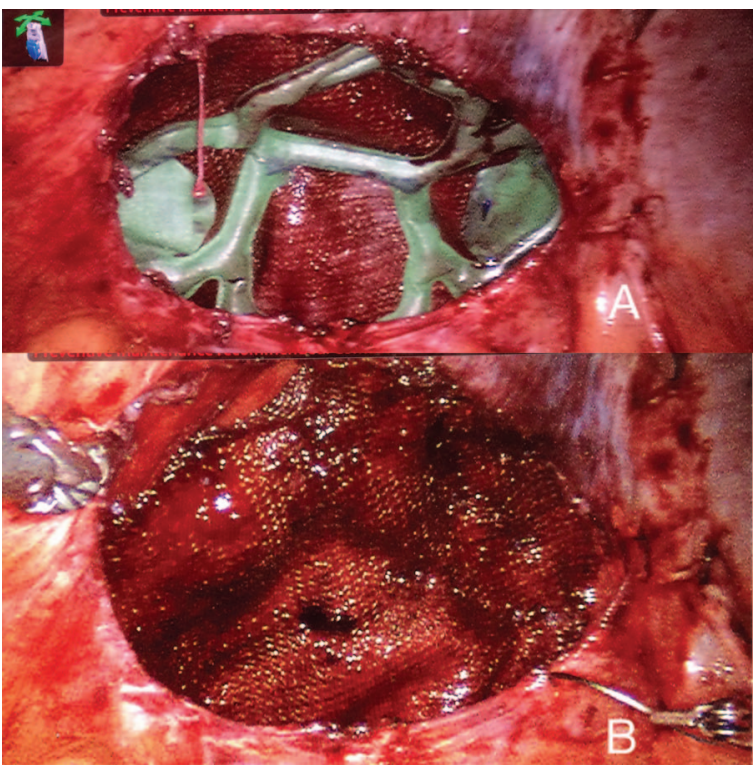

Figure 5. A polypropylene mesh $(11 \times 11 \mathrm{~cm})$ is placed in the preperitoneal space to cover the hernia defect. The ECHO PS system was used to center the mesh flat over the hernia defect $\mathbf{A}$. The balloon was deflated and removed before fixation of the mesh $\mathbf{B}$.

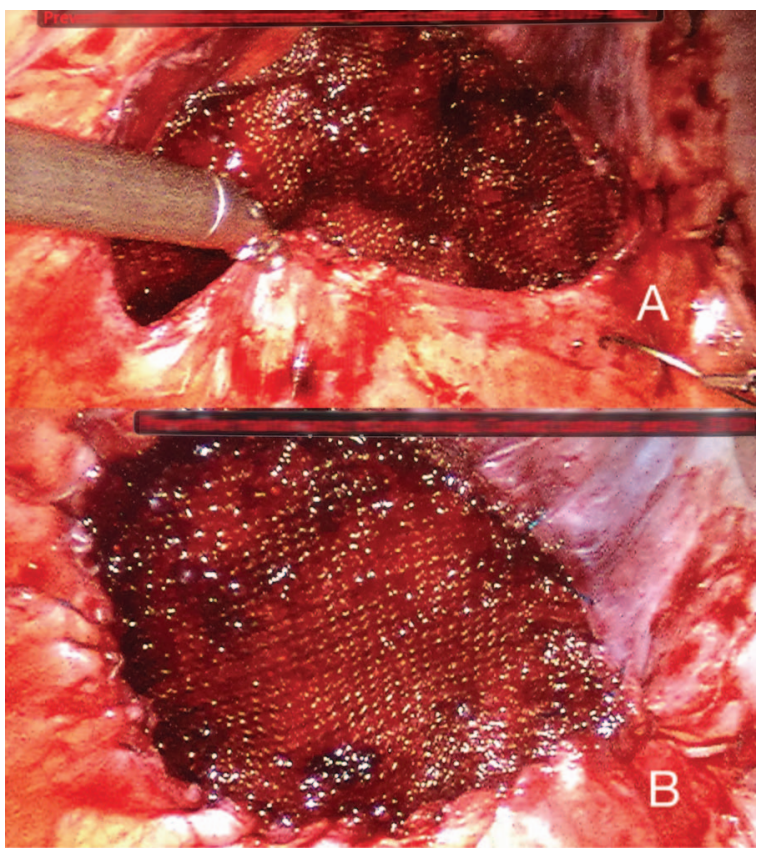

Figure 6. The peritoneum overlapped the edge of the mesh $\mathbf{A}$. A single running suture was used to fix the mesh in place, providing adequate coverage $\mathbf{B}$. more complex because of the various anatomic locations. There are no current guidelines for repair specific to nonmidline or lateral incisional hernias based on prospective data. These hernias are currently repaired with the same principles as are used for midline ventral hernias.

The robotic platform offers an expanded field of view and has the added benefit of wristed movements, which allows the surgeon to perform intra-abdominal suturing, with fixation of the mesh to the peritoneum and/or posterior fascia. 9 This method allows the surgeon to avoid using tacks or transabdominal sutures for suture fixation, which are often cited as the culprit for laparoscopic postoperative pain. ${ }^{10}$ The ergonomic features of the robot also give the surgeon the opportunity to create a peritoneal flap for preperitoneal mesh placement to prevent meshrelated intra-abdominal complications and reduce the risk of migration and lateral detachment, which are often cited as the cause of recurrence. ${ }^{11,12}$

To date, there are no case reports on the use of the robotic platform to repair a nonmidline ventral hernia from a hand-assist site. We used the guidelines and principles set forth for repair of midline ventral hernias and literature on lateral hernia repair to guide us in our repair. We were able to close the defect primarily, after raising musculofascial flaps, to recreate the abdominal wall contour. We were able to provide adequate mesh coverage beyond the defect and secure the mesh in a sublay fashion with a running intra-abdominal suture. We included the peritoneum in our fixation to cover the edge of the mesh, as this is often cited as the area of recurrence. Although the approach is feasible, prospective studies are needed to determine whether the robotic platform confers long-term benefits for patients who present with HALN incisional hernia repairs compared to standard laparoscopic repair.

\section{References:}

1. Cobb WS, Carbonell AM, Snipes GM, et al. Incisional hernia risk after hand-assisted laparoscopic surgery. Am Surg. 2012;78: 864-869.

2. Strup SE, Hubosky SG, Trabulsi EJ, McGinnis DE, Diamond SM, Gomella LG. Complications of hand-assisted laparoscopic nephrectomy: a review of 118 consecutive cases at a single institution. J Urol. 2002;167(4 suppl):168 Abstract 674.

3. Allison N, Tieu K, Snyder B, Pigazzi A, Wilson E. Technical feasibility of robot-assisted ventral hernia repair. World J Surg. 2012;36:447-452. 
4. Troxel SA, Das S. Incisional hernia following hand-assisted laparoscopic surgery for renal cell cancer. JSLS. 2005;9:196-198.

5. Pezeshk RA, Pulikkottil BJ, Bailey SH, et al. An evidencebased model for the successful treatment of flank and lateral abdominal wall hernias. Plast Reconstr Surg. 2015;136:377385.

6. Petro CC, Orenstein SB, Criss CN, et al. Transversus abdominis muscle release for repair of complex incisional hernias in kidney transplant recipients. Am J Surg. 2015;210:334-339.

7. Sauerland S, Walgenbach M, Habermalz B, Seiler C, Miserez M. Laparoscopic versus open surgical techniques for ventral or incisional hernia repair. Cochrane Database of Systematic Reviews. March 16, 2011.

8. Earle D, Roth S, Saber A, et al. Guidelines for Laparoscopic Ventral Hernia Repair. SAGES Guidelines Committee, July 2016.
9. Ballantyne, GH, Hourmont K, Wasielewski A. Telerobotic laparoscopic repair of incisional ventral hernias using intraperitoneal prosthetic mesh. JSLS. 2003;7:7-14.

10. Wassenaar E, Schoenmaeckers E, Raymakers J, Van der Palen J, Rakic S. Mesh-fixation method and pain and quality of life after laparoscopic ventral or incisional hernia repair: a randomized trial of three fixation techniques. Surg Endosc. 2010;24: 1296-1302.

11. Prasad P, Tantia O, Patle NM, Khanna S, Sen B. Laparoscopic ventral hernia repair: a comparative study of transabdominal pre-peritoneal versus intraperitoneal onlay mesh repair. J Laparoendosc Adv Surg Tech A. 2011;21:477-483.

12. Awad ZT, Puri V, LeBlanc K, et al. Mechanisms of ventral hernia recurrence after mesh repair and a new proposed classification. J Am Coll Surg. 2005;201:132-140. 